\title{
Microstructural features in carbonates from Antarctic micrometeorites: Effective tools for analyzing the evolution of small Solar System bodies
}

Elena Dobrica $^{1}$, Kenta Ohtaki ${ }^{2}$ and Cecile Engrand ${ }^{3}$

${ }^{1}$ University of Hawai'i at Manoa, United States, ${ }^{2}$ University of Hawaii at Manoa, Honolulu, Hawaii, United States, ${ }^{3}$ Université Paris-Saclay, Orsay, France

Carbonates are excellent candidates to study the secondary processes that modified the primitive materials that formed in the Solar System. They have the potential to provide us a high-fidelity record of environmental conditions and the timing of their formation [1-6]. Furthermore, they have been commonly identified in carbonaceous chondrites $(<2 \mathrm{vol} \%)$, though they are rare in dust particles [1-6]. Recently, centimeters-thick, roughly meter-long veins of carbonates were identified in outcrops at the surface of the asteroid Bennu [7]. Here we investigate the formation of carbonates identified in one Antarctic micrometeorite through detailed transmission electron microscopy (TEM) study of their chemical composition and crystallographic texture. Previous studies show that deviations in structure and stoichiometry from the ideal crystalline structure could help constrain the fluid conditions and record the evolution of the small Solar System bodies [8-10]. We use site-specific extraction techniques to investigate the mineralogy, texture, and chemical compositions of several electron transparent sections of one micrometeorite that contain carbonates.

One unmelted hydrated fine-grained Antarctic micrometeorites (H-FgMMs) (03-36-46) was analyzed in this study using extensive TEM techniques. The sample was collected from the pristine Concordia snow during the 2002 campaign [11]. The size of the particle analyzed in this study is $43 \times 112 \mu \mathrm{m}$, which corresponds to the whole particle collected from snow. The polished and carbon-coated petrographic section was first characterized by scanning electron microscopy (SEM) using backscattered electron imaging on a Helios 660 dual-beam focused ion beam SEM (FIB-SEM) instrument at the Advanced Electron Microscopy Center (AEMC) at UH Mānoa. Four electron transparent sections were prepared by the conventional in situ FIB technique. A platinum protective layer was deposited on top of the region of interest, first by electron beam deposition and then by ion beam deposition, to avoid gallium primary ion beam damage during the FIB sample preparation. The section was transferred to $\mathrm{Cu}$ transmission electron microscopy (TEM) half grids with a micromanipulator. The final ion milling of the $2 \mu \mathrm{m}$ thick section to electron transparency was carried out with the sample attached to the TEM grid. The final thinning stages were performed at $2 \mathrm{kV}$ with a current of $72 \mathrm{pA}$. Each FIB section was studied using various TEM techniques, including scanning transmission electron microscopy (STEM) imaging, nanodiffraction, and energy-dispersive X-ray spectroscopy (EDS). All imaging and analysis were carried out at $300 \mathrm{kV}$ using the Titan G2 analytical (S)TEM at AEMC. Crystalline phases were identified by electron nanodiffraction and EDS. Nanodiffraction was carried out with an accelerating voltage of $300 \mathrm{kV}$, a camera length of 295 $\mathrm{mm}$, and a convergence angle of $0.1-0.3 \mathrm{mrad}$.

TEM studies of the dolomite grains $\left[\mathrm{Ca} 0.9-1 \mathrm{Fe}^{2+} 0.1-0.2 \mathrm{Mg} 0.8-0.9 \mathrm{Mn}^{2+}{ }_{0.1-0.2}\left(\mathrm{CO}_{3}\right) 2\right]$ show a complex microstructure as indicated by the presence of modulations (Fig. 1). These modulations appear as parallel lamellae alternating dark/light contrast when imaged with a small convergence angle (0.1-0.3 mrad). In this study, we focused on one particular cluster composed of two crystals, which contains modulations affecting the entire crystal (Fig. 1). The interface between the two crystals (white dashed line Fig. 1a) in the dolomite cluster displays a moiré pattern formed by the superimposition of two periodical structures (Fig. 1). The two crystals show an orientation mismatch of a relative rotation angle of $\sim 4^{\circ}$ between lattice 
fringes. Additionally, the EDS analysis indicates that these modulations correspond to local compositional fluctuations in manganese (up to 1.4 at.\% Mn, Fig. 1b-c).

The microstructural features identified in the dolomite grains from the H-FgMM analyzed could help us to decipher the growth conditions and mechanisms that could have modified these crystals [8]. Despite numerous efforts to relate modulations to either compositional variations or strain, their origin is still debated. Previous studies indicate that they could arise from cation site ordering or slight displacements, or both [12]. Our TEM study shows that dolomites are extensively strained, display moiré patterns at the interface between crystals, and present large compositional variations. The presence of these microstructural features could suggest both (1) growth processes during a periodic variation in fluid composition and (2) shock deformation. Carbonates with such complex microstructures were identified previously in the Martian meteorite, Allan Hills 84001 [9-10]. These microstructures were interpreted as a shock feature [9-10]. Although the micrometeorite analyzed in this study does not show any evidence of shock, the presence of these microstructural features could indicate that carbonates may potentially record minor shock processes. Further studies will be carried out in other carbonates from chondrites and dust particles. The goal is to understand their origin and identify if any shock disturbance of the crystal lattice could generate errors in the measured $\mathrm{Mn}-\mathrm{Cr}$ isochrons or reset the $\mathrm{Mn}-\mathrm{Cr}$ system in some primitive components. 

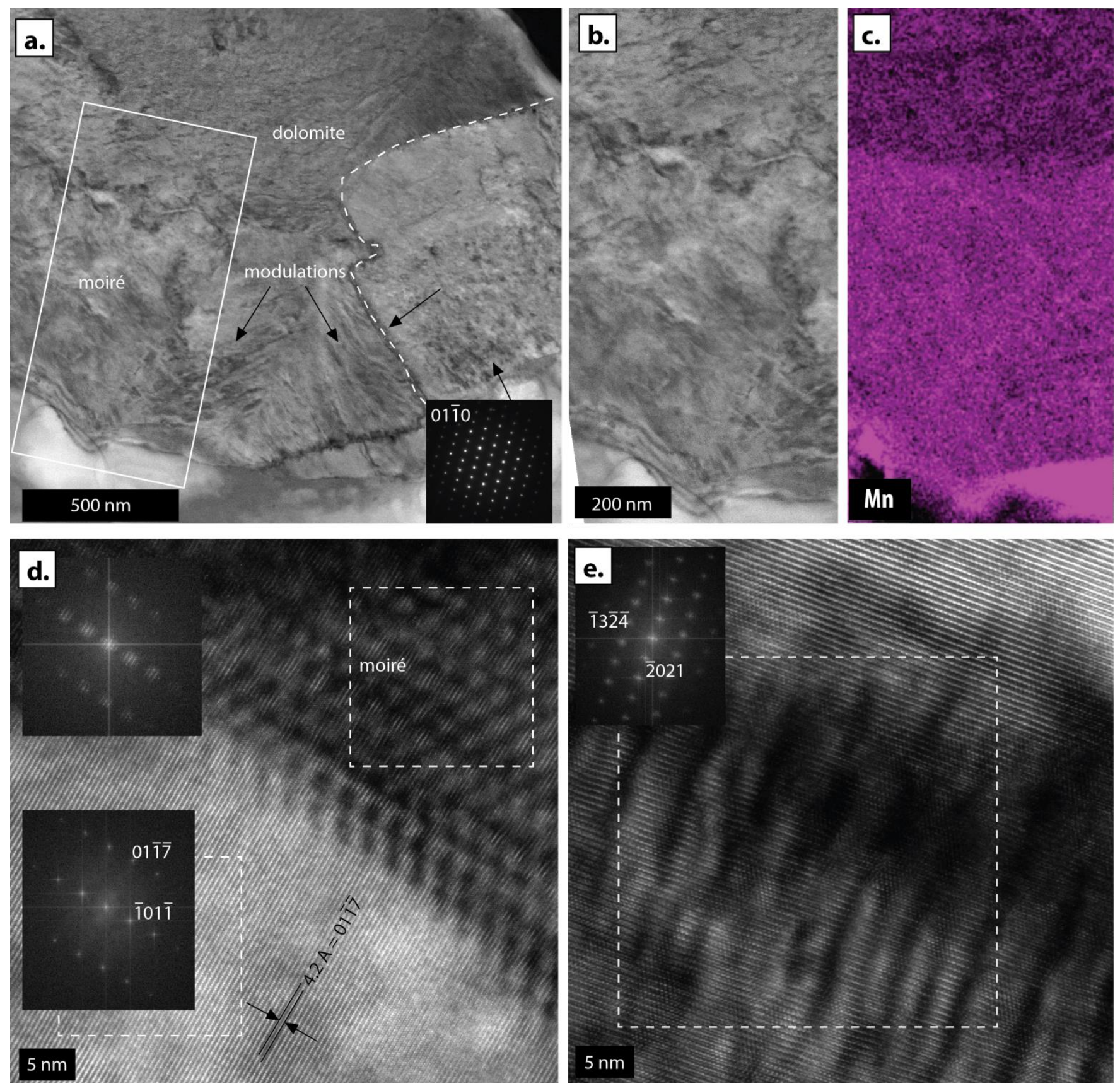

Figure 1. Figure 1. a) Bright-field (BF) TEM micrograph showing the complex microstructure of a cluster of dolomite crystals from the hydrated fine-grained Antarctic micrometeorites (H-FgMMs, 03-36-46). The dolomite cluster is composed of two crystals (the white dashed line shows the crystals interface) with different crystallographic orientations. The white box outlines the area shown in Fig. 1b-c. b-c) Brightfield (BF) TEM and EDS map of manganese showing the compositional variations (up to 1.4 at.\% Mn) of one dolomite grain. d-e) High-resolution TEM images of (d) moiré fringes at the grain boundary at the grain boundary and (e) the modulation in the center of the grain. Fast Fourier Transform (FFT) patterns of the HR images are shown. The FFTs show the pattern resulted from the moire structure (d - top) and one dolomite crystal close to the interface (d - bottom), and the modulation structure (e). This moiré FFT pattern ( $\mathrm{d}$ - top) exhibits peaks originating from the two lattices and the moiré peaks. 


\section{References}

[1] Dobrică E. et al. Meteorit. Planet. Sci. 54 (2019) 1973-1989.

[2] Alexander C. M. O’D. et al. Meteorit. Planet. Sci. 50 (2015) 810-833.

[3] Fujiya W. et al. Earth and Planetary Science Letters 362 (2013) 130-142.

[4] Tyra M. et al. GCA 77 (2012) 383-395.

[5] de Leuw S. et al. GCA 73 (2009) 7433-7442.

[6] Lee M. R. et al. GCA 144 (2014) 126-156.

[7] Kaplan H. H. et al. Science 370 (2020) 6517.

[8] Reeder R. J. Rev. in Mineral. 27 (1992) 381-424.

[9] Barber and Scott Meteorit. Planet. Sci. 41 (2006) 643-662.

[10] Brearley A. J. Meteorit. Planet. Sci. 38 (2003) 849-870.

[11] Duprat J. et al. Advances in Space Research 39 (2007) 605-611.

[12] Baronnet A. Rev. in Mineral. 27 (1992) 231-288.

Work supported by NASA Emerging Worlds grants NNX17AE25G to ED. 